\title{
An interview with
}

\section{Lucia Helena Soares Cevidanes}

- Dentistry Graduate, Federal University of Goiás, 1989.

- MSc in Orthodontics, Methodist Institute for Higher Education, 1994.

- PhD in Oral Biology, University of North Carolina at Chapel Hill, 2003.

- Assistant Professor, Department of Orthodontics, University of North Carolina at Chapel Hill.

- Diplomate, American Board of Orthodontics.

- Reviewer of the American Journal of Orthodontics and Dentofacial Orthopedics, Angle Orthodontist, Journal of Dental Research, European Journal of Oral Sciences, World Journal of Orthodontics, Orthodontics and Craniofacial Research, International Journal of Oral Maxillofacial Surgery, and Dentomaxillofacial Radiology.

- Thomas M. Graber Award of Special Merit by the American Association of Orthodontists, 2004.

- B. F. and Helen Dewel Award for best clinical article published in 2005 in the American Journal of Orthodontics and Dentofacial Orthopedics.

- Teaching Award by the American Association of Orthodontics Foundation in 2008 and 2009

It gives me great pleasure to conduct an interview with Professor Lucia Cevidanes, an example of humbleness, courage and determination. Born in Caratinga, Minas Gerais, she attended dentistry at the Federal University of Goiás and earned a Masters Degree in Orthodontics at UMESP, where she was faculty member for four years. After setting up a private practice in Santo André/SP, she decided to pursue her dream of earning a PhD abroad, which she accomplished at one of the most prestigious research centers in Orthodontics and Orthognathic Surgery worldwide. Building on a clinical sample she had tenaciously put together in Brazil, she entered the world of diagnostic imaging to undertake an award-winning research project. Ultimately, her outstanding contributions led her to a position as Faculty Member of the Department of Orthodontics at UNC, where she develops some of the most stimulating research projects in today's literature. Coordinating a research team comprised of American, European and Brazilian collaborators in experiments that make use of three-dimensional diagnosis, Prof. Cevidanes spends her time on a wide range of activities, such as lectures in different countries, clinical and theoretical teaching activities at Graduate and Masters courses in Orthodontics, participation in an interdisciplinary group devoted to the treatment of craniofacial anomalies while still maintaining a clinical orthodontic practice at the institution. Married to Larry, who is also a professor at UNC in the field of psychology, she has two daughters, Teresa and Angelina, who she enjoys taking for a stroll down Franklin Street, in Chapel Hill, on week-ends. They also travel on vacation to visit friends in Connecticut or family on their farm in Minas Gerais State, Brazil. 
What is your outlook on the dissemination of Cone-Beam Computed Tomography (CBCT) among clinicians and what knowledge and equipment are necessary before it can be used routinely in diagnosing, planning and evaluating orthodontic, orthopedic and surgical treatment? Ary dos Santos-Pinto

Several hurdles must be overcome before CBCT is used routinely in clinical orthodontics:

a) Laying down guidelines to determine which cases benefit from additional clinical information to justify its higher cost and increased radiation dose to patients. The Board of Trustees of the American Association of Orthodontists (AAO) and American Academy of Oral and Maxillofacial Radiology (AAOMR) has appointed a council committed to having these guidelines ready by the end of 2010. It includes the following orthodontists: Dr. Carla Evans (Univ. of Chicago), Dr. Martin Palomo (Case Western University), Dr. Kirt Simmons (Arkansas Children's Hospital) and Dr. Lucia Cevidanes. Radiologists in the group are led by Dr. William Scarfe (Univ. of Louisville), Dr. Mansur Ahmad (Univ. of Minnesota) and Dr. John Ludlow (Univ. of North Carolina). The guidelines are "to be reviewed every three years as scientific evidence builds up in the literature". ${ }^{1}$

b) Validation and development of threedimensional analysis software. Current versions of commercial software are still fraught with limitations and require monthly updates. Moreover, the accuracy of the tools employed in these programs has not yet been scientifically validated.

c) Absence of standard population data to support diagnostic analysis. Issues related to identifying anatomical landmarks in traditional cephalometric analysis have been regarded as a major source of errors in determining the key craniofacial measurements. In 3D, this problem is further compounded by the fact that many anatomical landmarks are poorly defined in one of the three planes of space. For example, the gonial point is located on a curve, which makes determining its location in the vertical plane an error-prone process.
Given the increasing use of 3D CBCT images, a recurring question emerges: should we use them in all cases or only in selected cases? Alexandre Motta

In my opinion, these images should be used in selected cases. For example, Class I malocclusion cases without tooth impaction do not justify the use of Cone-Beam tomography.

For which clinical procedures or clinical cases would you consider it essential to request computed tomography in orthodontic practice? Liliana Maltagliati

The guidelines to determine which cases can benefit from CBCT clinical information will be laid down by the joint efforts of the AAO and AAOMR. Not only which cases can benefit from CT, but also on which occasions or how often this radiographic follow-up procedure is indicated. Comparisons using population standards and two-dimensional (2D) cephalometric representations fail to address many issues pertaining to diagnosis and mechanisms of treatment response and growth. Planning treatment for the following orthodontic problems, in particular, can be potentially enhanced by 3D diagnostic information: Skeletal anchorage with mini-plates (Fig 1), dental impaction or eruption failure, patients with maxillomandibular discrepancy in any of the three planes of space (transversal - asymmetries; vertical - open/deep bite; anteroposterior - skeletal Class II and III), and temporomandibular disorders (TMDs).

As regards image acquisition, do you believe different devices such as the NewTom and iCAT can provide comparable quality images, or would the differences compromise the serial, longitudinal superimposition? Could differences in image acquisition with patients lying or sitting affect these assessments, especially those of the airways for the purpose of diagnosing nasal, nasopharyngeal and oropharyngeal obstructions? Ary dos Santos-Pinto 
Depending on the software used for viewing, image voxel size needs to be standardized so that images acquired with different equipment, such as the NewTom and i-CAT, are comparable. If proper care is not exercised when performing $\mathrm{CBCT}$ in centric occlusion, differences in image acquisition with patients lying or sitting could affect, in particular, not just the assessment of the airways and facial soft tissues but mandibular posture as well. Currently, all our images are acquired using a thin wax bite in centric occlusion. Additionally, images acquired using the NewTom display more noise, especially in the image periphery, often compromising the quality of $3 \mathrm{D}$ surface models (Fig 2).

After years studying imaging, initially with magnetic resonance, investigating the effects of functional appliances on TMJ, and then later with computed tomography, how important do you really think these diagnostic imaging methods are for treating TMDs? Liliana Maltagliati

In my view, imaging diagnostics and TMD treatment are two areas where considerable research is still needed. TMD treatment is still narrowly focused on alternatives to minimize patient discomfort and pain. Despite many theories conducted beyond the field of Orthodontics and Oral Rehabilitation, the etiology of TMD involves facial myalgias and neuralgias for which CBCT imaging diagnostics would not be indicated. A clinical diagnosis using the parameters defined by the Diagnostic Criteria for TMD (RDC/TMD criteria $)^{2}$ is indicated before patients are referred for Cone-Beam CT (Fig 3). ${ }^{3}$

Would surface bone remodeling (resorption and apposition) pose a limitation to 3D image superimposition? Daniela Garib

Not at all. However, the techniques of 3D image superimposition to assess surface bone remodeling (resorption and apposition) must not make

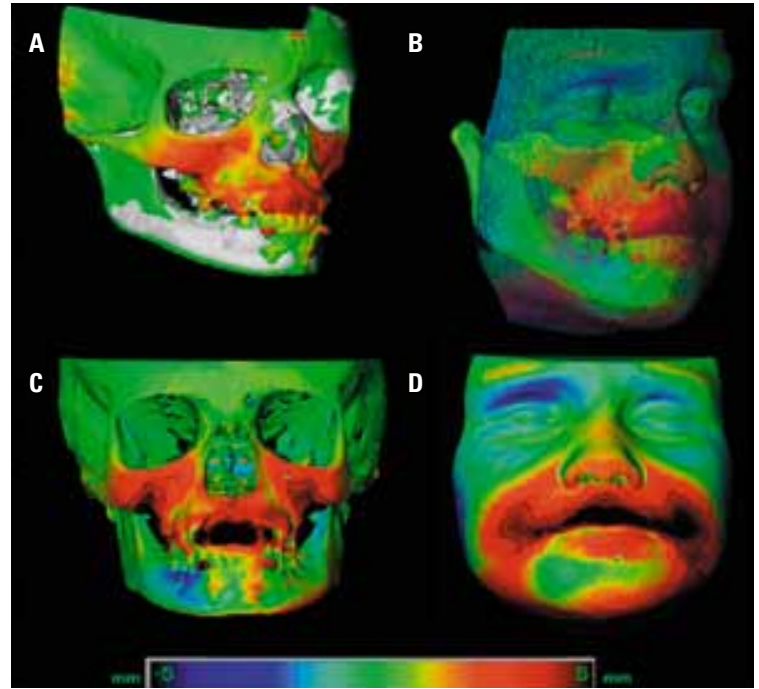

FIGURE 1 - Superimpositions on the anterior cranial fossa to assess relative growth and response to orthopedic treatment with skeletal anchorage in the maxilla and mandible. Anterior displacement of the midface (in red).

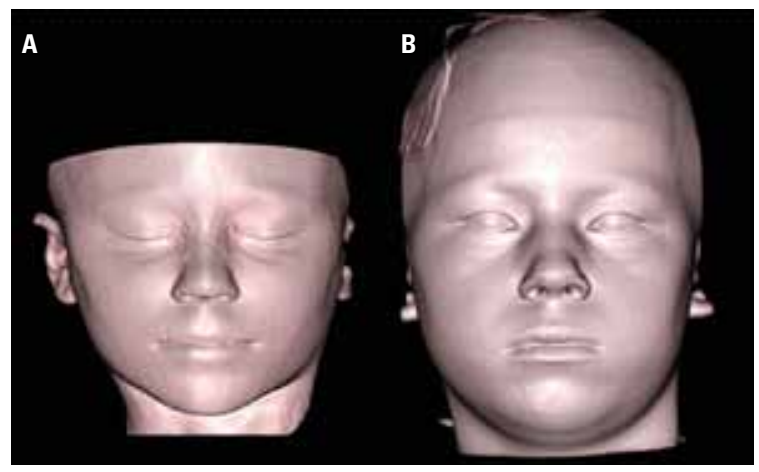

FIGURE 2 - Visualization of soft tissues in the faces of two patients with NewTom (A) and i-CAT (B) scans. Note that both scans show acceptable quality image with control of surface artifacts, very common in ConeBeam technique. Also note the increased definition of the surface scan produced with the i-CAT scanner.

use of adjacent structures as reference but rather regional superimposition (Fig 4). The study of bone remodeling in the mandible, for example, must use stable structures during mandibular growth, as in Bjork's 2D studies. In cases of mandibular surgery, this is complicated because the mandible is changed by surgery, so any "best fit" technique has a bias toward evaluating postoperative remodeling. 


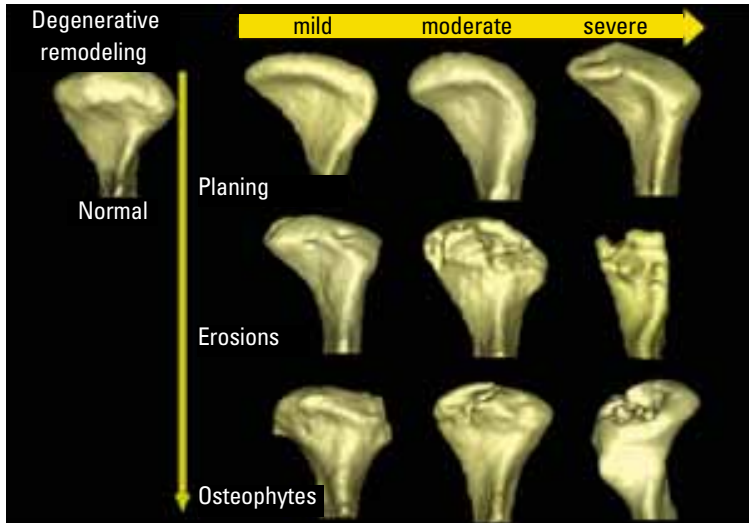

FIGURE 3 - Degenerative remodeling of the mandibular condyle in patients with TMD.

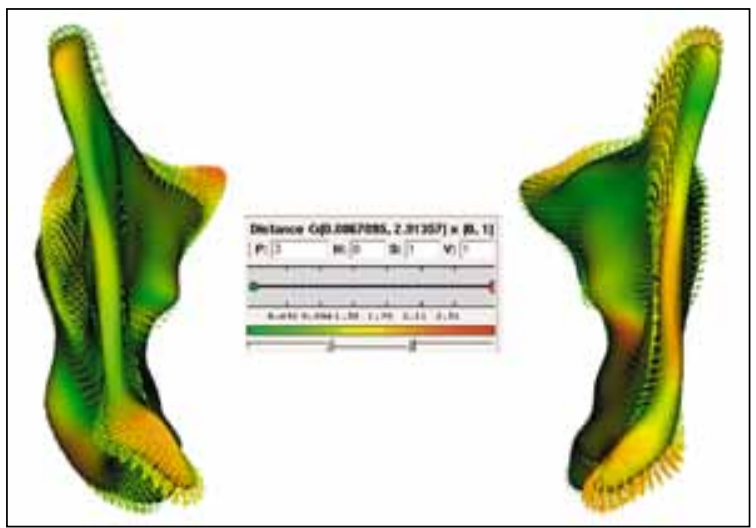

FIGURE 4 - New methods of 3D superimposition on the mandible, showing bone remodeling vectors in a patient with idiopathic condylar resorption.

Can an examination of study models in orthodontics be performed directly on the images of the dental arches, thus eliminating the need to take impressions of the dental arches? Ary dos Santos-Pinto

The best reference on orthodontic study models performed directly on images of the dental arches is the work published by Dr. Gwenn Swennen. ${ }^{4}$ As explained in detail in her article, it requires more than one scan, and a well calibrated device in order to correct artifacts in the region of the brackets and restorations.
What are the main differences between commercial and free three-dimensional analysis software? Alexandre Motta

Commercial software provides clinicians with a more user-friendly interface. The major issue is price. Besides, as remarked in my reply to the first question above, despite the marketing appeal of impressive diagnostic images the accuracy of most commercial software tools has yet to be validated scientifically. The ongoing development of public domain software is supported by the National Institute of Health in the United States, but with research, not commercial purposes. Their focus is on improving the quality of image analysis and not just developing user-friendly software for use in routine clinical practice. Thus, this software can run better on Linux than on Windows or Mac, as their computer graphics programs are developed for the Linux operating system.

How do you envisage the transition of 3D superimposition techniques from the research universe to clinical practice? Daniela Garib

Firstly, the barriers I mentioned in my first answer regarding the routine use of $\mathrm{CBCT}$ in orthodontic practice need to be surmounted. 3D superimposition methods currently used in research must undergo considerable development before they are employed in clinical routine, thanks in large measure to a platform recently developed by the National Institute of Health in the United States, which incorporates several features from different imaging modalities, including CBCT, spiral scanning, magnetic resonance and ultrasound, as well as several analysis procedures for building 3D models, superimposition, visualization and quantification aimed at diagnosing and assessing treatment results.

As the use of CT in clinical research intensifies, we anticipate an increased potential for errors that can compromise outcome, especially 
in "before and after" studies, given the difficulty in reproducing cross sections in successive examinations. What precautions would you recommend to help researchers avoid errors in methodology? Liliana Maltagliati

I agree that this is a serious risk we will be facing, mainly due to a lack of knowledge and proper training in 3D analysis. Clinicians have a hard time understanding analyses that are not based on anatomical landmarks because they are mathematically more complex. In November 2009, a group of American professors led by Dr. Martin Palomo and Mark Hans, from Case Western University, held their second meeting, where they discussed the standardization of image superimposition techniques, and these discussions will continue throughout November 2010.

In light of your academic experience around the world as a researcher and lecturer, what major trends and future prospects do you see for the application of 3D technology in orthodontics? Alexandre Motta

The use of 3D images for diagnosis, treatment planning, surgical simulation, evaluation of orthodontic treatment and biomechanical results has aroused great interest and led to the development of research worldwide.

As a Brazilian orthodontist who plays a brilliant role as a researcher in one of the most prestigious research centers in the country that saw the birth of orthodontics, what are your views on Brazilian orthodontics today? Daniela Garib

Orthodontics in Brazil has been developing and keeping up to date and dynamic largely owing to the efforts of excellent researchers. I have also had the pleasure and privilege of keeping in touch and collaborating with teachers and students from several Brazilian institutions in the development of some major research projects.

\section{REFERENCES}

1. Atkins D, Eccles M, Flottorp S, Guyatt GH, Henry D, Hill S, et al. Systems for grading the quality of evidence and the strength of recommendations I: Critical appraisal of existing approaches. BMC Health Serv Res. 2004 Dec 22;4(1):38.

2. Ahmad M, Hollender L, Anderson Q, Kartha K, Ohrbach $\mathrm{R}$, Truelove EL, et al. Research diagnostic criteria for temporomandibular disorders (RDC/TMD): development of image analysis criteria and examiner reliability for image analysis. Oral Surg Oral Med Oral Pathol Oral Radiol Endod. 2009 Jun;107(6):844-60.

3. Cevidanes LH, Hajati AK, Paniagua B, Lim PF, Walker DG, Palconet $\mathrm{G}$, et al. Quantification of condylar resorption in temporomandibular joint osteoarthritis. Oral Surg Oral Med Oral Pathol Oral Radiol Endod. 2010 Jul;110(1):110-7.

4. Swennen GR, Mollemans W, De Clercq C, Abeloos J, Lamoral P, Lippens F, et al. A cone-beam computed tomography triple scan procedure to obtain a threedimensional augmented virtual skull model appropriate for orthognathic surgery planning. J Craniofac Surg. 2009 Mar;20(2):297-307. 


\section{Alexandre Trindade Motta}

- Adjunct Professor of Orthodontics, Fluminense Federal University (UFF).

- PhD, MSc and Specialist in Orthodontics, Rio de Janeiro State University (UERJ).

- Sub-coordinator, Specialization Program in

Orthodontics, UFF.

- Board member of the Brazilian Society of Orthodontics (SBO).

- Fellow-researcher, University of North Carolina at Chapel Hill (UNC).

\section{Ary dos Santos-Pinto}

- Adjunct Professor, Department of Child Dentistry/ Orthodontics, School of Dentistry, Araraquara (UNESP). - MSc and PhD in Orthodontics, Federal University of Rio de Janeiro (UFRJ).

- Postdoctoral Research, Baylor College of Dentistry, Dallas/Texas, USA.

- Full Professor, postgraduate courses in Dental

Sciences/Orthodontics, MSc and PhD levels (Unesp).

- Scientific advisor: Dental Press Journal of Orthodontics and Revista Clínica de Ortodontia Dental Press.

\section{Daniela Gamba Garib}

- Professor and PhD in Orthodontics, School of Dentistry of Bauru and Hospital for Rehabilitation of Craniofacial Anomalies, University of São Paulo.

- Assistant Editor of the Dental Press Journal of Orthodontics.

- MSc and PhD in Orthodontics, Federal University of Rio de Janeiro (UFRJ).

- Postdoctoral Research, Harvard School of Dental Medicine, Boston, USA.

\section{Liliana Maltagliati}

- MSc and PhD in Orthodontics, Rio de Janeiro Federal University (UFRJ).

- Coordinator, Specialization Program in Orthodontics, ABCD-SP.

- Program Coordinator, Orthodontic Treatment of Adults, CETAO - SP.
Contact address

Lucia Cevidanes - 201 Brauer Hall

School of Dentistry, UNC Chapel Hill - Orthodontics - CB \#7450

Chapel Hill, NC 27599-7450

Email: cevidanl@dentistry.unc.edu 